\title{
Therapieentscheidung bei frühem Lungenkarzinom personalisieren
}

\author{
Besonders ältere, inoperable Patienten mit nichtkleinzelligem Lungen- \\ karzinom (NSCLC) profitieren von einer stereotaktischen ablativen Strahlen- \\ therapie (SABR), allerdings ist das Ergebnis variabel. Niederländische \\ Strahlentherapeuten präsentieren ein Modell zur Personalisierung.
}

$\mathrm{D}$ as Amsterdamer Modell zur Prognose des 5-Jahres-Überlebens von Patienten mit frühem NSCLC, die mit SABR behandelt werden, basiert auf einer rekursiven Partitionierungsanalyse (RPA) und einem Nomogramm, das zusätzlich individuelle Faktoren berücksichtigt. Datensätze von 703 solcher Patienten wurden im Verhältnis 2:1 in eine Trainings- und eine Validierungskohorte geteilt. Bei der Analyse der TrainingsDatensätze wurden 21 Einzelparameter von Patienten, Behandlung und Tumor in das RPA-Modell integriert und mittels univariater und multivariater Modelle auf ihre Bedeutung für das Überleben untersucht. Faktoren, die im multivariaten Modell signifikant waren, gingen in das Nomogramm ein. Extern wurden RPA und Nomogramm an unabhängigen chirurgischen $(n=193)$ und SABRDatensätzen $(\mathrm{n}=543)$ validiert.

Die RPA unterschied zwei verschiedene Risikoklassen, basierend auf den Parametern Tumordurchmesser, Alter, WHOPerformancestatus und Carlson-Comorbidity-Index. Die Abgrenzung der Risikogruppen in den SABR-Datensätzen gelang so mäßig gut (c-Index 0,52-0,60), während die externe Validierung bei operierten Patienten wenig aussagekräftig war. Zusätzlich zu den RPA-Faktoren be- rücksichtigte das Nomogramm die Raucheranamnese. Dieses Modell war von hohem Aussagewert sowohl in der internen Validierung $\left(r^{2}=0,97\right)$ als auch bei externen SABR- $\left(\mathrm{r}^{2}=0,79\right)$ und chirurgischen Datensätzen $\left(\mathrm{r}^{2}=0,91\right)$.

Fazit: Das Amsterdamer Modell ist das erste extern validierte Prognose-Tool für das Gesamtüberleben zur individualisierten Entscheidungsfindung bei Patienten mit frühem NSCLC und SABR. Das Nomogramm war in SABR- und chirurgischen Kohorten aussagekräftig, das RPA nur in SABR-Datensätzen - offensichtlich handelt es sich um unterschiedliche Patientenkollektive. Friederike Klein

Louie AV et al. Predicting Overall Survival After Stereotactic Ablative Radiation Therapy in EarlyStage Lung Cancer: Development and External Validation of the Amsterdam Prognostic Model. Int J Radiat Oncol Biol Phys. 2015;93(1):82-90.

\section{NSCLC: Jeder Zehnte mit EGFR-Mutationen}

\section{Bei asiatischen Patienten mit nichtkleinzelligem Lungenkarzinom (NSCLC) kennt man die Zusammenhänge mit therapierelevanten EGFR-Mutationen recht gut. Erstmals wurde dies in der großen REASON-Studie bei Patienten in Deutschland untersucht.}

$\mathrm{D}$ ie mehr als 4.000 Patienten in der Registerstudie - aus insgesamt 149 Studienzentren, darunter 22 onkologischen Praxen - hatten ein NSCLC im Stadium IIIb oder IV. Sie waren weder

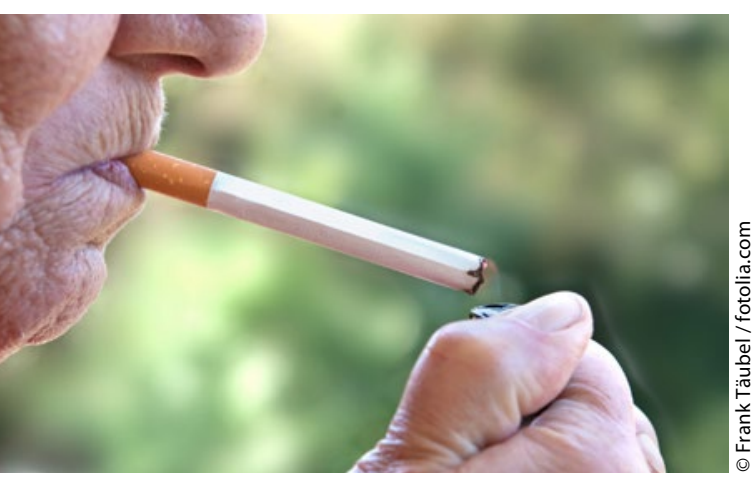

Raucher hatten seltener EGFRMutationen als Nichtraucher. für eine kurative Operation noch für eine Strahlentherapie geeignet, sondern Kandidaten für eine systemische Ersttherapie: abhängig vom Mutationsstatus, einer kombinierten Chemotherapie oder einem der in Deutschland zugelassenen Tyrosinkinasehemmer (TKI), Erlotinib oder Gefitinib. Die Mutationsanalysen erfolgten dezentral an nach der Qualitätssicherungsinitiative Pathologie (QuIP) zertifizierten pathologischen Instituten. Die Testung auf Mutationen in Exon 19 und Exon 21 waren bindend, Exon 18 und 20 optional. Primäres Ziel war es, die Verteilung des EGFR-Status erstmals in einer überwiegend weißen Patientenpopulation zu untersuchen sowie seine Korrelation mit klinischen Parametern.

Aktivierende EGFR-Mutationen wurden bei 10,3\% der Patienten nachgewiesen, bei $85,5 \%$ wurde keine Mutation ge- funden, bei 175 Patienten ließ sich der Status nicht sicher bestimmen.

EGFR-Mutationen wurden häufiger bei Frauen als bei Männern entdeckt (16,7 vs. $6,4 \%)$, häufiger bei Nichtrauchern als bei Rauchern ( 26,2 vs. $6,8 \%$ ) und häufiger bei Patienten mit einem Adenokarzinom im Vergleich zu anderen Histologien (13,1 vs. 3,8\%). Schließlich war die EGFR-Mutationsrate in Metastasen höher als in Primärtumoren (16,5 vs. $9,3 \%)$.

Fazit: Aktuelle Leitlinien empfehlen vor der NSCLC-Ersttherapie mit einem TKI eine Mutationsanalyse. In der REASONStudie aber erhielten $43,4 \%$ der Patienten mit positivem Testergebnis keine solche Therapie. Weil die Mutationen außer bei Frauen, Nichtrauchern und Patienten mit Adenokarzinom auch bei anderen Patientengruppen vorkommen, ist es wichtig, alle Patienten mit NSCLC im Spätstadium zu testen. Peter Leiner

Schuette W et al. EGFR mutation status and first-line treatment in patients with stage III/IV non-small cell lung cancer in Germany: an observational study. Cancer Epidemiol Biomarkers Prev. 2015;24(8):1254-61. 